\title{
Correspondence
}

\section{BAG3 protein expression in melanoma metastatic lymph nodes correlates with patients' survival}

\author{
L Guerriero ${ }^{1}, \mathrm{~K}_{\text {Chong }}{ }^{2}$, R Franco ${ }^{3}$, A Rosati ${ }^{\star, 1,4}, \mathrm{~F}$ De Caro ${ }^{5}$, M Capunzo ${ }^{5}$, MC Turco $^{4,5}$ and DSB Hoon ${ }^{2}$ \\ Cell Death and Disease (2014) 5, e1173; doi:10.1038/cddis.2014.143; published online 10 April 2014 \\ Subject Category: Cancer
}

\section{Dear Editor,}

Patients diagnosed with stage III melanoma with clinically evident regional lymph node (LN) metastasis can differ significantly in disease outcome. This significant variability (24-70\% 5-year survival) to some extent is likely due to known prognostic factors but is also related to tumor heterogeneity in the expression of specific genes. ${ }^{1}$ The first-line therapy is generally represented by therapeutic LN dissection (TLND), and the current approaches to stratify patients with this stage of disease predominantly involve clinical and histological parameters ${ }^{2}$ such as number of positive LN and extracapsular extension. However, these factors do not completely account for the disease outcome and the identification of novel molecular biomarkers could help to better categorize the heterogeneity of stage III melanoma tumors.

Recently, BAG3 protein was shown to act as a pro-survival factor in several tumor types including melanomas. ${ }^{3,4}$
Indeed, in an in vivo model of human melanoma xenografts, we showed that BAG3 is able to interfere with the HSP70mediated delivery of the IKK- $\gamma$ subunit of IKK complex to proteasome, thereby sustaining NF- $\kappa \mathrm{B}$ activation and inhibiting tumor cell apoptosis; BAG3 silencing resulted in a significant reduction of tumor growth and in prolonging animal survival. ${ }^{4}$ BAG3-positive staining was observed in a subset of primary human melanomas and metastasis, and higher levels of BAG3 expression were registered in metastases compared with primary tumors. ${ }^{5}$ Because of the significant contribution of the protein to melanoma cells' survival in experimental models, ${ }^{4}$ we decided to investigate a possible correlation between BAG3 expression in regional LN metastasis and survival of patients who underwent TLND.

We analyzed BAG3 expression in a tissue microarray (TMA) of clinically palpable LN metastases of stage III melanomas. ${ }^{6}$
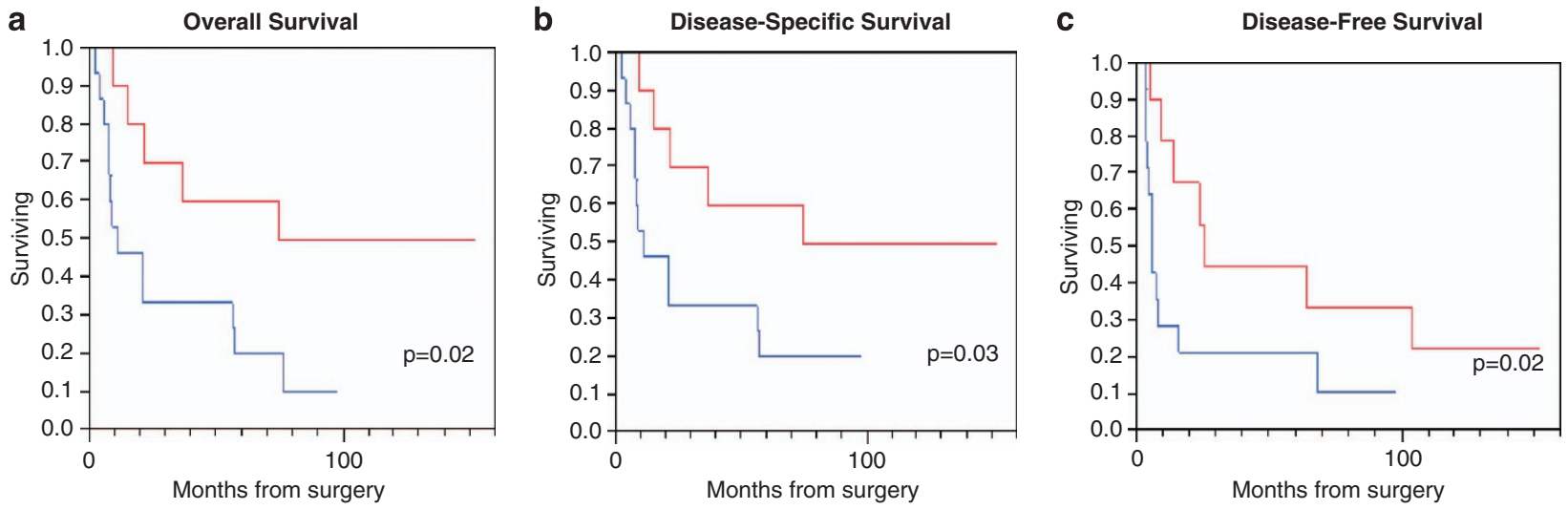

$$
\begin{aligned}
& \text { - BAG3 high positive } \\
& \text { - BAG3 low positive }
\end{aligned}
$$

Figure 1 Analysis of BAG3 correlation of patients' survival with 2-3 positive LN with OS (a), DSS (b) and DFS (c). Samples in the TMA were analyzed by immunohistochemistry using the anti-BAG3 mAb AC-1 (BIOUNIVERSA s.r.l., Fisciano, Italy). The difference between BAG3 low-positive and BAG3 high-positive samples was assessed as previously described. ${ }^{7}$ Survival analyses were performed by Kaplan-Meier curves using commercially available software (release 12; SigmaPlot, Systat Software Inc., San Josè, CA, USA)

\footnotetext{
${ }^{1}$ Department of Pharmacy (DIFARMA), University of Salerno, Fisciano, Italy; ${ }^{2}$ Department of Molecular Oncology, John Wayne Cancer Institute, Santa Monica, CA, USA; ${ }^{3}$ U.O.C. Anatomia Patologica, Istituto Nazionale Tumori Fondazione G. Pascale, Naples, Italy; ${ }^{4}$ BIOUNIVERSA S.r.l, University of Salerno, Fisciano, Italy and ${ }^{5}$ Department of Medicine and Surgery, University of Salerno, Baronissi, Italy

${ }^{*}$ Corresponding author: A Rosati, Cellular Biochemistry Lab N ${ }^{\circ}$ 19, Division of Biomedicine 'Arturo Leone', Department of Pharmacy, University of Salerno, Via Giovanni Paolo II, 132, 84084 Fisciano, Italy. Tel: + 39089 968171/18; Fax: + 39089 968775; E-mail: arosati@ unisa.it
} 
This TMA comprised tumor-negative LNs as controls and LN metastases from 149 patients, of which 76 patients had a poor prognosis (recurrence or death $<2$ years) and 73 remained disease-free for $>5$ years. ${ }^{6}$ We decided to analyze BAG3 positivity results in subgroups of patients divided for one of the major prognostic factors, such as the number of positive LN. Subgroups analyzed were individuated into: patients with only 1 positive $\mathrm{LN}(N=46)$, patients with $2-3$ positive $\mathrm{LN}(N=35)$, and the latter one represented by patients with $\geqslant 4$ positive $L N$ $(N=48)$. In the subgroup of patients with 2-3 positive $L N$, we found a significant correlation between BAG3 positivity and patients' overall survival (OS), disease-specific survival (DSS) and disease-free survival (DFS) from surgery. Indeed, using the cut-off $>25 \%$ of BAG3-positive cells, we found that in BAG3 low-positive samples OS corresponded to a median of 52.8 months, whereas in BAG3 high-positive this was 29.2 months $(P=0.02$; Figure 1a); similar results were observed for DSS $(P=0.03$; Figure $1 \mathrm{~b})$ and DFS $(P=0.02$; Figure 1c). Although the results have to be confirmed in a larger set of patients, we found an interesting correlation between BAG3 expression and prognosis, in agreement with similar findings in another tumor type, that is, pancreatic adenocarcinoma. $^{8}$

In conclusion, these results identify a subgroup of stage III melanoma patients, that is, patients with 2-3 positive LNs, whose clinical behavior is influenced by the expression of the anti-apoptotic BAG3 protein. We suggest that BAG3 analysis on LN biopsies could therefore contribute to prognosis and patient stratification for specific therapeutic approaches.

\section{Conflict of Interest}

BIOUNIVERSA s.r.l., which produces anti-BAG3 antibodies, provided them free of charges for this study. AR and MCT are shareholders of the company BIOUNIVERSA that provided some of the used antibodies. The remaining authors declare no conflict of interest.

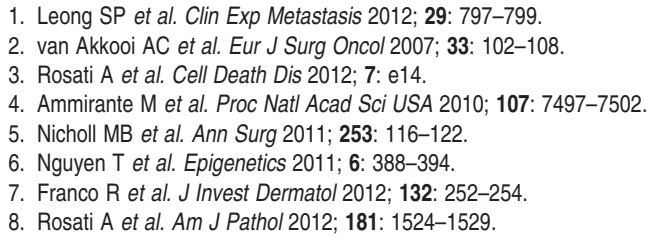

(c) (i) (2) Cell Death and Disease is an open-access journal published by Nature Publishing Group. This work is licensed under a Creative Commons Attribution-NonCommercialShareAlike 3.0 Unported License. The images or other third party material in this article are included in the article's Creative Commons license, unless indicated otherwise in the credit line; if the material is not included under the Creative Commons license, users will need to obtain permission from the license holder to reproduce the material. To view a copy of this license, visit http://creativecommons.org/licenses/ by-nc-sa/3.0/ 